\title{
Automatic Generation of Caricatures with Multiple Expressions Using Transformative Approach
}

Chien-An Lai and Wen-Hung Liao

\{g9202,whliao\}@cs.nccu.edu.tw

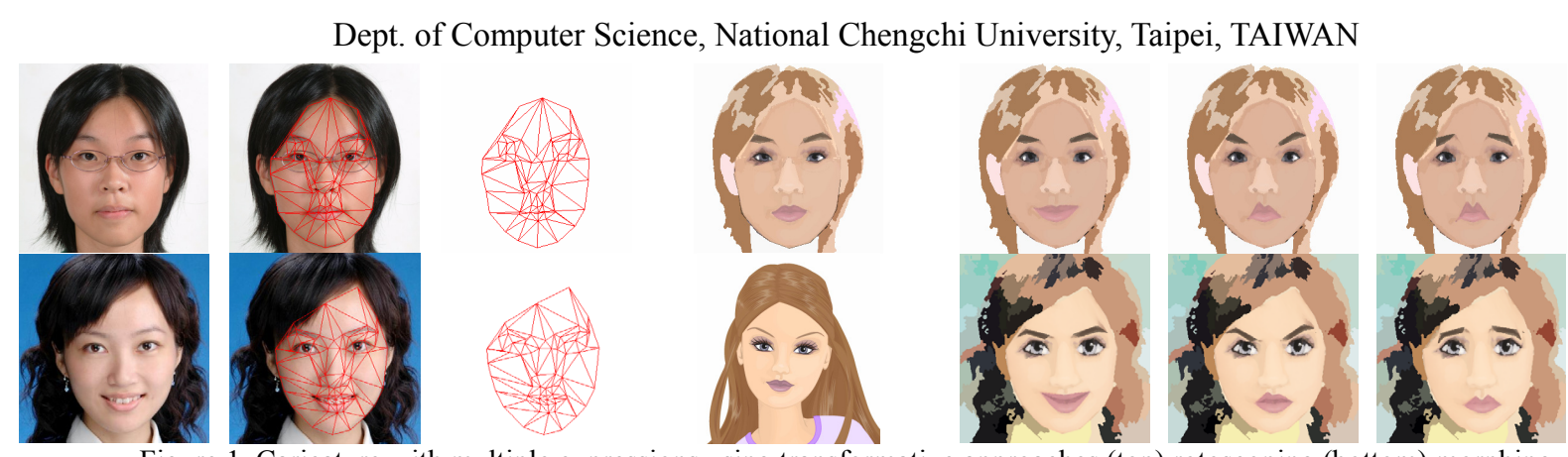

Figure 1. Caricature with multiple expressions using transformative approaches (top) rotoscoping (bottom) morphing

\section{Introduction}

The proliferation of digital cameras has changed the way we create and share photos. Novel forms of photo composition and reproduction have surfaced in recent years. In this sketch, we present an automatic caricature generation system using transformative approaches. By combing facial feature detection, image segmentation and image warping/morphing techniques, the system is able to generate stylized caricature using only one reference image. The system can also produce multiple expressions by controlling the MPEG-4 facial animation parameters (FAP). A major strength of our method is that the synthesized caricature bears a higher degree of resemblance to the real person than traditional component-based approaches.

\section{Caricature Generation}

First, a collection of reference cartoons with different styles has to be built. A frontal-view photo is then presented as the source image. Using active appearance model (AAM), we can locate important facial components and the corresponding mesh, as shown in Fig. 1.

The caricature can be generated using two different approaches: rotoscpoing or image morphing. In rotoscoping, we combined contour detection, mesh generation, histogram specification and mean-shift algorithm to obtain a simplified region-based representation of the face with user-defined color palette, as shown in the top row of Fig. 1.

In image morphing, we defined 8 components, comprising a total of 66 control points, to describe the facial configuration. Once the corresponding triangular mesh between the reference cartoon and the input image is identified, barycentric coordinate is employed to speed up the morphing process. Typical results are depicted in the bottom row of Fig. 1. Aside from manual selection of the reference cartoon, the system can also provide recommendation based on shape similarity using Fourier descriptor. Blending of styles can be easily accomplished using polymorph technique [1]. The weight is inversely proportional to the distance between the input shapes and the respective reference caricatures.

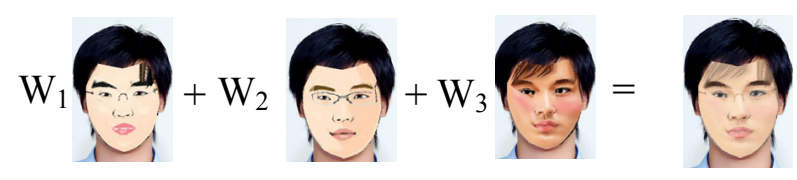

Figure. 2 New styles generated with polymorph technique

\section{Synthesis of Multiple Expressions}

We decompose the archetypal expression profile defined in [2] to arrive at a reduced set of facial animation parameters (FAP) for 2D caricatures. Using facial component action definition, the FAP can be set to manipulate the 66 facial definition points (FDP) directly to synthesize different expressions. In addition to the 6 basic emotion types identified by Ekman [3], we have also defined 12 profiles according to popular emoticons, as shown in Fig. 3.
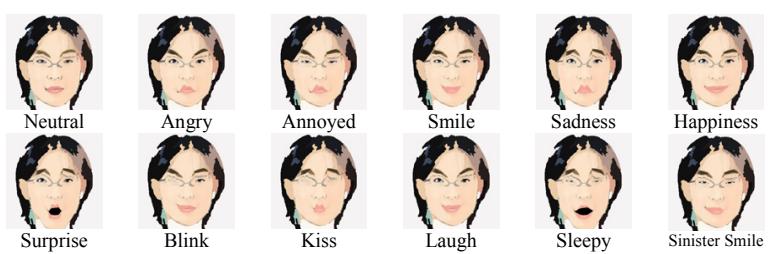

Figure. 3 Expression mimicking popular emoticons

\section{Conclusion}

We have demonstrated an automatic caricature generator that is capable of synthesizing personalized cartoons with multiple expressions using only one reference image. New styles can be easily created by blending existing samples.

\section{References}

[1] S. Lee, G. Wolberg, and S. Y. Shin, "Polymorph: Morphing Among Multiple Images", IEEE CG\&A, Vol.18, 1998.

[2] K. Perlin "Layered Compositing of Facial Expression", ACM SIGGRAPH 97 Technical Sketch.

[3] P. Ekman, "Facial Expression and Emotion", Am Psychologist, Vol.48, 199 